\title{
Devaluation: a dynamical mechanism for a naturally small cosmological constant
}

\author{
Katherine Freese* and James T. Liu \\ Michigan Center for Theoretical Physics, University of Michigan, Ann Arbor, MI 48109-1120 \\ Douglas Spolyar \\ Physics Department, University of California, Santa Cruz, CA 95060
}

\begin{abstract}
We propose a natural solution to the cosmological constant problem consistent with the standard cosmology and successful over a broad range of energies. This solution is based on the existence of a new field, the devaluton, with its potential modeled on a tilted cosine. After inflation, the universe reheats and populates the devaluton's many minima. As the universe cools, domain walls form between different regions. The domain wall network then evolves and sweeps away regions of higher vacuum energy in favor of lower energy ones. Gravitation itself provides a cutoff at a minimum vacuum energy, thus leaving the universe with a small cosmological constant comparable in magnitude to the present day dark energy density.
\end{abstract}

The discrepancy between the observed vacuum energy density of the universe today and the much larger value expected from the standard model of particle physics is known as the cosmological constant problem. At the same time, however, recent observations of Type IA Supernovae 1, 2] indicate that the universe is accelerating. One possible explanation of the "dark energy" driving this acceleration is that it is due to a cosmological constant of $(0.003 \mathrm{eV})^{4}$; we take this as an upper limit on the allowed value. However, this value of the cosmological constant is unnaturally small in particle physics.

Unnatural cancellations of zero point energies for particles and for vacuum energies generated at phase transitions are required to one part in $10^{120}$ so that the sum of all these terms is small enough to agree with the observations. The hope is sometimes voiced that a fundamental quantum theory of gravity will resolve this problem, but such a theory must in fact give rise to a cosmological term (say, at the Planck scale) which is precisely cancelled by all lower energy contributions (e.g. at the electroweak scale and below) to one part in $10^{120}$. Thus the cosmological constant problem is essentially a difficulty of physics at very 'low' energies, suggesting that its solution will come instead from new physics manifest at low energies. One possibility invokes compensating fields whose vacuum energy dynamically adjusts to cancel the large value described above which arises from particle physics.

In this letter, we propose a dynamical solution to the cosmological constant problem (including any contributions generated at phase transition) involving the postinflation evolution of a domain wall network. What is required is a model with a sufficiently rich potential which has nearly degenerate minima (separated by barriers of appropriate height) spanning a range of both negative and positive values of vacuum energy. After a conventional stage of inflation followed by reheating, different

\footnotetext{
*Electronic address: ktfreese@umich.edu

${ }^{\dagger}$ Electronic address: jimliu@umich.edu

‡Electronic address: dspolyar@physics.ucsc.edu
}

patches of the universe fall into different minima, and a domain wall network is thus formed.

At this stage, the universe contains many different regions with different vacuum energies separated by domain walls. However, a domain wall separating two regions with different vacuum energies will feel a pressure driving it to move rapidly into the region of higher energy. As a result, the domain walls end up sweeping away regions of high positive energy, thus driving the universe to lower and lower vacuum energy.

It is of course important to this scenario that the universe does not end up falling into a state with large regions of negative vacuum energy. We will show below that this does not happen, as gravity differentiates between positive and negative energies. The essential feature here is similar to the Coleman and de Luccia result that tunneling into an anti-de Sitter vacuum is greatly suppressed in the presence of gravity. In the present case, however, this argument involves the evolution of domain walls (as opposed to instantons) interpolating between different vacuum states. As a result, the universe naturally evolves to a final (metastable) state with the potential sitting at the minimum closest to zero energy.

Given an appropriate potential, this final value of the energy density could be made on the order of $\left(10^{-3} \mathrm{eV}\right)^{4}$ in agreement with observational measurements of the dark energy. Ideally, one would like to see this value come out naturally as part of the solution to the cosmological constant problem. However, at present we are unable to claim success in this regard, and simply view this result as imposing a requirement on the landscape of the minima of the potential.

We denote this process of domain wall evolution driving the universe to a state of nearly zero vacuum energy by "devaluation", the process of moving towards lower and lower values of the cosmological constant. As a concrete realization of this devaluation model, we may consider a system with two scalar fields, the first an inflaton $\psi$ which drives inflation and the second the devaluton $\Phi$ which is responsible for the domain wall network. Of course, the overall evolution of the universe is 
governed by the nature of the coupled potential $V(\psi, \Phi)$ (which includes the vacuum energy of the standard particle physics sector). However, with an appropriate separation of scales, we may treat the evolution of the universe in two sequential stages, the first being inflation and the second devaluation. Thus we take

$$
V(\psi, \Phi)=V_{\mathrm{inf}}(\psi)+V_{\mathrm{dev}}(\Phi)
$$

The energy scales of the inflaton can be anything from Grand Unified (GUT) scale down to $10 \mathrm{MeV}$, as long as reheating above nucleosynthesis is successful. The devaluton too can have a wide range of energy scales: $V_{\text {dev }}$ can become negative, but its magnitude is roughly bounded by the energy scale of the inflaton. In particular, we absorb any uncontrolled contribution to the vacuum energy into the inflaton potential $V_{\mathrm{inf}}$.

Inflation Field. In the inflationary stage, the vacuum energy dominates the energy density, and the scale factor of the universe expands superluminally to solve the horizon, flatness and monopole problems of standard cosmology [3]. The inflaton potential may come from either a tunneling field or a rolling field. In either case the important features are sufficient inflation followed by reheating, with the potential at the end of inflation at some positive value $\geq 10 \mathrm{MeV}$. It is this remaining amount of vacuum energy that is removed by the devaluation process. We emphasize that the details of the inflaton are not important for our devaluation idea; in the next few paragraphs we present an example of a possible inflaton field.

For a dynamical solution to the cosmological constant problem, we cannot simply postulate the absolute minimum of the inflaton potential $V_{\text {inf }}$ to be zero. Thus this requirement that $V_{\text {inf }}$ ends up at a sufficiently small positive value at the end of inflation is not a trivial one. It turns out, however, that this requirement is naturally satisfied in the recently proposed chain inflation model [4], which resolves the problems of old inflation while retaining the idea of tunneling fields dominating the energy density of the universe.

In chain inflation, one considers a potential $V_{\text {inf }}$ that has many minima of different vacuum energies (above and below zero) with barriers separating the different minima. The field quantum starts out in a high energy minimum and quantum mechanically tunnels through a series of barriers from higher to ever lower minima. The universe inflates by a fraction $N_{\text {stage }}<1 / 3$ of an $e$-fold while sitting in each minimum, adding up to at least 60 $e$-folds from all the tunneling events $\left(N_{\text {tot }}>60\right)$. The number of minima that the field must go through must be at least 200 .

A simple model for the chain inflation potential is a cosine with a linear tilt

$$
V_{\mathrm{inf}}(\psi)=V_{0}\left[1-\cos \frac{\psi}{v_{1}}\right]-\alpha \psi .
$$

Due to this tilt, any uncontrolled amount of vacuum energy may be absorbed by a shift in $\psi$. Of course, to be realistic, this potential ought to be cut off at an arbitrary energy below zero. However the details of the cutoff are unimportant as the field never tunnels completely to the minimum. While the energy scales of this potential can be anywhere from the GUT scale down to $10 \mathrm{MeV}$, we assume the width, barrier height and tilt of the potential are all roughly comparable. For example, if the energy scale $\sim 1 \mathrm{GeV}$, in the absence of any tuning, there will typically be a minimum at $V_{\mathrm{inf}} \sim \frac{1}{2} \mathrm{GeV}$ and another at $V_{\text {inf }} \sim-\frac{1}{2} \mathrm{GeV}$. The inflaton then starts in a high energy minimum and subsequently undergoes a series of tunneling events until it stops tunneling and gets stuck at the last positive energy minimum.

The reasons why the universe does not enter a negative energy vacuum are twofold. Firstly, Coleman and de Luccia [5] studied tunneling in the thin wall limit and concluded that, for large enough bubbles, tunneling is suppressed to AdS vacua. They also found that, if tunneling were to take place, the result would be a negative curvature universe collapsing to a Big Crunch. Subsequently, Banks [6] generalized these results to claim that decays into spaces of negative cosmological constant do not occur in consistent theories of quantum gravity as the requisite instantons for tunneling into AdS simply cannot exist.

The second argument for why the field gets stuck at the last positive vacuum, rather than decaying to AdS, is as follows. At each stage of tunneling, bubbles are produced of the lower energy vacuum. Because $N_{\text {stage }}<1 / 3$, the bubbles percolate at each stage. However, in the earliest stages of chain inflation, the interiors of the bubbles are still dS so they cannot thermalize. Only once the field has tunneled many times and is approaching Minkowski space can the interiors really thermalize. At this stage one must consider the tunneling in the presence of radiation. This process has previously been considered by Affleck and de Luccia 7], who found that the presence of particles can either enhance or reduce the rate of decay of the false vacuum. By properly adjusting the coupling, the tunneling rate can be made very slow. However this suppression is only relevant in the present case for $V_{\mathrm{inf}}$ near zero since that is where the radiation becomes thermalized. Hence this mechanism can be used to allow tunneling at high energies but to delay tunneling into AdS vacua. We note that rolling fields may alternatively drive the inflation, as long as the vacuum at the end of inflation reheats to a positive energy value.

Devaluation Field. At this stage, the inflaton field has served its purpose in driving inflation (and reheating in the last few stages of chain inflation). After inflation, the universe is radiation dominated and proceeds through its ordinary evolution, including primordial nucleosynthesis. With $V_{\text {inf }}$ sitting in a positive energy minimum $\geq 10 \mathrm{MeV}$, it is now the rôle of the devaluton to further drive the vacuum energy to near zero. This is the most important feature of our model. To do so, we may take 
a periodic devaluton potential of tilted cosine form

$$
V_{\mathrm{dev}}(\Phi)=V_{0}\left[1-\cos \frac{N \Phi}{v}\right]-\eta \cos \left[\frac{\Phi}{v}+\gamma\right] .
$$

This potential is bounded and has many minima with large barriers in between. The bumps in the potential are present at any temperature, but only become important once the temperature drops to the value of the barrier height. Furthermore, this potential is similar to that of the QCD axion, where the periodicity in the first term is due to instanton effects and the tilt in the second term can be produced with a soft-breaking term (or by coupling to an additional scalar field). Such a small tilt, crucial to our model, is then natural as it arises from soft breaking of a symmetry.

Defining $\theta=N \Phi / v$, the potential has $N$ minima at $\theta=2 \pi n$ where $n=0,1,2, \ldots, N-1$ and we take $N$ to be a large number. The barrier height can be expressed in terms of the devaluton mass $m$ as

$$
V_{0}=m^{2} v^{2} / N^{2}
$$

and the wall tension is

$$
\sigma=8 m v^{2} / N^{2} .
$$

The second term tilts the potential and thereby breaks the degeneracy between the minima, where the energy difference between minima is roughly

$$
\epsilon \sim 2 \pi \eta / N,
$$

and $\gamma$ is a phase. Due to the tilt in the potential, the different minima of the potential have different energies, ranging from $-\eta$ to $+\eta$. To ensure the possibility of zero vacuum energy, we must take $|\eta|$ to be larger than $V_{\text {inf }}$ at the end of chain inflation. Furthermore, the energy difference $\epsilon$ must be chosen on the order of $\left(10^{-3} \mathrm{eV}\right)^{4}$ to be consistent with present observations. This demonstrates that $N$ must be very large, on the order $10^{10}$ or more.

At the end of inflation, but at temperatures much larger than the devaluton barrier height, the barriers go unnoticed and the universe populates all values of $\Phi$ (with bias towards lower energies in the tilt). However, once the temperature drops down to the value of the barrier height $V_{0}$, the barriers become important. Different patches of the universe fall into different minima of the devaluton potential separated by domain walls. Note that $\Phi$ does not tunnel from one local minimum to the next as the barrier height between minima is too large. However, because the potential is tilted, the different minima of the potential have different energies, and this provides a bias driving the domain wall evolution.

This domain wall evolution was previously investigated by Sikivie [8] in the context of the QCD axion. In that context the tilt in the potential was introduced to eliminate excess domain walls by driving them away. One way to see this is to note that domain wall networks with nondegenerate vacuum states mainly evolve due to two competing effects: a surface tension that acts to straighten the walls and a volume pressure due to the energy difference between the minima. The latter force causes the walls to move into the region of higher energy, thereby making such regions smaller. This behavior has been shown to be rather varied and interesting [9]. Provided the dynamical timescale for this evolution is sufficiently rapid, the domain walls thus evolve to eliminate regions of higher energy vacuum in favor of lower energy ones. In this way, the universe, which consists of a patchwork of regions with different values of the devaluton field (sitting in different minima of the devaluton potential), is driven to a very low value of the cosmological constant.

As for the timescale, Larsson et al. [10] have studied the evolution of non-degenerate domain wall networks to see how quickly they disappear. They consider two sources of instability: first the pressure due to the energy difference between minima, and second any bias in the original probability distribution that prefers one vacuum over the other. In our case, there is the obvious bias incurred by the fact that we are considering a radiation dominated universe: a Boltzmann distribution favors the lower energy vacuum. However, near the minimum of the potential the distribution should be rather flat. As shown in [10], even a tiny statistical bias between two minima plays an important role in eliminating the less favored minimum. They performed numerical simulations to show that, when the typical domain size has grown to a critical radius $R>R_{c} \sim \sigma / \epsilon$, the wall energy density decays exponentially fast. The pressure acts very rapidly (faster than on cosmological timescales) to eliminate the high energy vacua in favor of lower energy ones.

Furthermore, the case of adjacent regions of different vacuum energy has previously been carefully considered by Cvetič et al. 11, 12, 13]. They studied the case of a single domain wall separating two regions of different vacuum energy which can be either positive energy (dS), zero energy (Minkowski), or negative energy (AdS). In their study, there was no radiation in the system, merely vacuum. They explicitly computed the dynamics of two regions separated by a domain wall by making an ansatz for the metric on two sides and connecting them via the Israel matching conditions on the domain wall boundary.

This devaluation process, whereby domain walls sweep away regions of high positive energy and drive the universe to vacuum energy near zero (or to the current value of the dark energy), provides a natural solution to the cosmological constant problem. We note that, unlike other proposed solutions to the cosmological constant problem, reheating is successful in this model. Here radiation was produced at the end of inflation, leading to the usual radiation and matter dominated evolution of the universe. In fact radiation is a critical element in the devaluation process. Because the universe is radiation (or matter) dominated (rather than vacuum dominated), the horizon grows in time. Hence neighboring regions which were initially causally disconnected become causally connected as time goes on. Thus domain walls sweep across ever larger regions of the universe, so that larger and 
larger patches of the universe are driven to the same low value of the vacuum energy. The radiation of course is also a key element in ending up with a universe that looks like ours which contains matter and radiation.

Negative Energy Vacua. There is of course an important issue that remains to be addressed, namely whether or not the universe falls into minima of the devaluton field with values of vacuum energy that are below zero. Clearly we would not want to evolve into a universe with a large negative value of the cosmological constant. However we show that this does not happen. Thus the devaluation process stops near Minkowski space rather than driving the universe to a large negative vacuum.

We first recall from above that the results of Coleman and de Luccia [5] indicate that the universe will not enter a negative energy state at the end of the chain inflation period. Hence the devaluation stage will be initiated with all regions of space at positive energy. While the devaluton may explore regions where its potential $V_{\mathrm{dev}}$ is negative, the thermal energy will always be sufficient to lift the energy above zero. Domain walls do not form until the universe cools. However, once they do form, they will divide the universe into separate regions with $\Phi$ in different wells of the potential. If one or more of these regions have negative vacuum energy, then they would presumably end up dominating the universe. However, there are important gravitational constraints on the formation of domain walls separating regions of negative vacuum. In particular, the radius of curvature of such domain walls must become ever larger for more negative energy vacua. Once this radius of curvature becomes infinite, the domain wall can no longer form. Hence this provides a cutoff in how negative the energy can be on one side of the wall. Any patches of space with excessive negative energy cannot become isolated from adjacent regions through domain wall formation. Thus they will continue to exchange radiation with the surrounding regions until they are brought back up to a non-negative (or only slightly negative) amount of energy. The result is that it is impossible for the universe to fall into regions with a cosmological constant more negative than a critical value.

To understand the cutoff on domain wall formation, we turn again to the work of Cvetič et al. 11, 12, 13. which provided a careful investigation of domain walls in gravity. In particular, these authors found that the vacuum domain walls fall in three classes depending on their value of wall tension $\sigma$. These three classes are determined by whether their wall tension is smaller, larger, or equal to a value $\sigma_{\text {static }}$ determined by

$$
8 \pi G \sigma_{\text {static }}=2\left(\alpha_{1} \pm \alpha_{2}\right),
$$

where $G$ is Newton's constant, subscripts 1 and 2 refer to the two regions on the two sides of the wall, and the value of the cosmological constant on either side is $\Lambda_{i}=-3 \alpha_{i}^{2}$.

The first class, know as extreme walls, occurs when $\sigma=\sigma_{\text {static }}$. Extreme walls are planar static walls, corresponding to supersymmetric configurations, and can only interpolate between Minkowski and Ads (Type I) or AdS and AdS [Type II or III depending on the sign in (7)]. The second class, where $\sigma>\sigma_{\text {static }}$, are know as nonextreme walls. These correspond to the interesting situation where observers on both sides of the domain wall see the wall as receding away from the observer. One can think of this situation as both observers living inside ever-expanding bubbles. Finally, the third class is the ultra-extreme case where $\sigma<\sigma_{\text {static }}$. Here, an observer in the higher energy region sees a bubble of lower energy sweeping towards her, so that in the end only the lower energy region remains.

As ought to be evident, devaluation domain walls fall into the ultra-extreme category where lower energy regions push aside regions of higher energy. In this case the wall tension $\sigma$ is related to the wall curvature $\beta^{2}$ according to

$$
8 \pi G \sigma / 2=\left(-\Lambda_{1} / 3+\beta^{2}\right)^{1 / 2}-\left(-\Lambda_{2} / 3+\beta^{2}\right)^{1 / 2} .
$$

It is perhaps more illuminating to invert this for the wall curvature. The result is

$$
\beta^{2}=\frac{4 \pi^{2} \sigma^{2}}{m_{p l}^{4}}+\frac{\Lambda_{1}+\Lambda_{2}}{6}+\frac{\left(\Lambda_{2}-\Lambda_{1}\right)^{2} m_{p l}^{4}}{64 \times 9 \pi^{2} \sigma^{2}} .
$$

While $\beta^{2} \geq 0$, we note that the limit $\beta^{2} \rightarrow 0$ corresponds to taking the bubble radius $R \sim \beta^{-1}$ to infinity. Since a larger radius is of course impossible, we obtain a bound on how negative the vacuum energy can be. Setting $\beta^{2}=$ 0 in (9) yields a minimum possible value for the average of the cosmological constants

$$
\left(\Lambda_{1}+\Lambda_{2}\right)_{\min }=-\frac{3}{2}\left[\frac{16 \pi^{2} \sigma^{2}}{m_{p l}^{4}}+\frac{m_{p l}^{4}(\Delta \Lambda)^{2}}{16 \times 9 \pi^{2} \sigma^{2}}\right],
$$

where $\Delta \Lambda=\left|\Lambda_{2}-\Lambda_{1}\right|$, and any two neighboring regions have values of the cosmological constant that differ by $\Delta \Lambda=8 \pi \epsilon / m_{p l}^{2}$. This indicates that there is a minimum negative value of the cosmological constant; anything more negative precludes the formation of the domain wall in the first place. For example, by taking $\epsilon=\left(10^{-3} \mathrm{eV}\right)^{4}$ (on the order of the present day cosmological constant), we obtain

$$
\begin{gathered}
\left(\rho_{v 1}+\rho_{v 2}\right)_{\min }=-\left(10^{-3} \mathrm{eV}\right)^{4}\left(\frac{\sigma}{(10 \mathrm{MeV})^{3}}\right)^{-2} \\
\times\left(\frac{\epsilon}{\left(10^{-3} \mathrm{eV}\right)^{4}}\right)^{2}
\end{gathered}
$$

where $\rho_{v i}=\Lambda_{i} / 8 \pi G$. The universe is then left with a small cosmological constant comparable in magnitude to the present day dark energy density. The bound here has negative energy density while the dark energy has a positive value.

Cvetič et al. have shown that Eqs. (7) and (8) can also be derived by simple energy arguments. They define a gravitational (Tolman) mass density $\rho_{G}=\rho+3 p$. For a 
negative vacuum $\rho_{G}=+2\left|\rho_{v a c}\right|$, i.e., attractive. Hence, in the competition between pressure and tension describing the dynamics of the domain walls, the AdS side exhibits an attractive "force" on the wall. This additional attraction tends to make small AdS regions disappear [30]. For more negative AdS, an ever larger radius of curvature is required in order for the AdS region to be stable or to push aside its higher energy neighbors. Above we have computed the case of infinite radius to find the most negative allowed AdS region.

We should note, however, that the results of Refs. 11, 12, 13 depend on simplifying assumptions that may not hold here. Firstly, they considered asymptotically dS, Minkowski and AdS spaces, while our patches may be quite small. In addition, the universe in our model has a radiation component that was not included in their studies. A complete treatment taking into account these additional issues would be an important topic for future study. We also note that we have not considered the case of $\beta^{2}<0$, which corresponds to imaginary radius for the domain wall. By analogy with the work of [5], this case may lead to singularities and has not yet been examined.

At least up to these caveats, we find that regions of the universe with large negative cosmological constant are never allowed to come into existence. When different patches of the universe fall into different minima of the potential, they are constrained to fall into minima with cosmological constant no more negative than allowed by Eqs. (10) or (11). Domain wall evolution then proceeds naturally to lower the cosmological constant to zero (or just below zero).

Other Issues. One might yet worry that some horizonsized regions of the universe would fall into very high energy minima of the devaluton potential and subsequently get trapped without devaluating down to Minkowski space. If they inflated, these de Sitter dominated regions could end up eating up a large portion of the universe, so that the average spot in the universe would remain in a very high value of vacuum energy, much higher than is observed. However, it can be demonstrated that these potentially dangerous patches do not in fact cause any problems. Within each horizon sized region (which grows during radiation domination), there are likely to be many regions, one of which has a low energy vacuum that takes over the higher energy ones (as the domain walls sweep them away). In addition, the population of high energy vacua is Boltzmann suppressed: Larsson et al [10] have shown that a tiny difference in population (one part in $10^{8}$ ) is sufficient to dynamically drive a two-state system to the lower energy vacuum. As these high energy regions are driven to lower energies, we do not need to resort to anthropic arguments to explain why we are not in such a region [31].

The model presented here shares similar features [including the potential (3)] with an earlier proposal by Abbott [19] for dynamically reducing the vacuum energy. In his model, the universe begins at a large value of the vacuum energy and then descends down the potential with decreasing vacuum energy. At low enough vacuum energy the barriers between minima become important and the field must tunnel from one minimum to the next lower one. The tunneling rate has been computed in the presence of gravity by Coleman and de Luccia [5], and as mentioned above has been shown to become slower as the field approaches AdS. Abbott chose the parameters of his potential so that the universe ends up with a small positive cosmological constant, as the lifetime for decay to negative cosmological constant is longer than the age of the universe. However, Abbott's model fails in that it gives an empty universe with no radiation or matter content. Attempts to revive this model include [20], but the problem of an empty universe persists. Other variants include 21] and 22]. The key similarity between Abbott's model and ours is that it relies upon the ability of gravity to differentiate between $\mathrm{dS}$ and AdS vacua. Gravity knows when the system has reached zero energy and makes it hard to go lower.

Although our devaluton potential is the same as the one used by Abbott, the major differences between Abbott's model and ours are as follows. First, we have no tunneling between minima of the devaluton (our second field); instead domain walls sweep aside higher positive energy vacua in favor of lower energy ones. Second, we do have reheating and radiation in our model due to an additional inflaton field at higher energies.

We briefly discuss what type of particle the devaluton could be. One constraint is that it cannot destroy predictions of Big Bang Nucleosynthesis (BBN). Possibilities include (i) a thermal relic more massive than $1 \mathrm{MeV}$, (ii) a particle lighter than $1 \mathrm{MeV}$ that fell out of equilibrium before BBN, or (iii) a particle that couples to the standard model only gravitationally. In the latter case, the interactions between the standard and hidden sectors are so weak that the two sectors decouple at the Planck scale with the two temperatures then evolving independently. Such gravitationally coupled devalutons can be treated as 'shadow matter' as in [23, 24, 25]. Candidates for the devaluton also exist in string theory such as (i) axions and (ii) moduli, which naturally arise in string compactifications and which may have instanton generated corrections to their potentials.

Domain walls produce temperature anisotropies in the microwave background of magnitude $\delta T / T \sim G \sigma L[26]$ where $L$ is the distance between walls. Since the walls in our model disappear via devaluation, these anisotropies are harmless. The largest contribution is from horizon sized walls with $L \sim t_{d}$ where $t_{d}$ is the universe age at the time the walls disappear, roughly at $T_{d} \sim$ $V_{0}^{1 / 4}$. Taking $\delta T / T \leq 10^{-5}$ from observations, we find $\sigma \leq(1 \mathrm{MeV})^{3}\left(T_{d} /\left(10^{-3} \mathrm{eV}\right)\right)^{3 / 2}$, which may constrain our model. Structure formation with unstable domain walls has been considered by [27, 28].

We briefly mention another possibility for bringing the cosmological constant to a small value. One could have two tunneling fields which both chain inflate [e.g. potentials like those of Eq. (2)], in lieu of an inflaton and a 
devaluton. If one pursues to the extreme the argument that there can be no tunneling to an AdS vacuum [6], one could postulate a tunneling chain inflaton (leading to reheating) followed by a second chain tunneling field with tilt $\sim 10^{-3} \mathrm{eV}$ that simply stops tunneling when the potential reaches $V=+10^{-3} \mathrm{eV}$. Then the current value of the vacuum energy would be the dark energy and the cosmological constant problem would be resolved. However, we find this solution too simple and have proposed the devaluation mechanism as more robust and interesting.

Summary. We have discussed a dynamical solution to the cosmological constant problem. After inflation and reheating, different patches of the universe fall into different nearly degenerate minima of the devaluton potential separated by domain walls. We have seen that the domain walls drive the system to the minimum closest to zero energy. As long as devaluation begins after the electroweak scale (the barrier heights of the potential are lower than the electroweak scale), the potential including all known contributions to the cosmological constant is driven to a very small value, in agreement with observations. The concrete example described above employed two scalar fields, one for inflation and the other for devaluation. However, we note that the basic idea can also work for a larger number of fields. For example, there could be a devaluton at each order of magnitude in mass scale: e.g. if inflation ends at the GUT scale, as the temperature drops, first the devaluton with barrier height $10^{15} \mathrm{GeV}$ becomes important, then the one with barrier height $10^{14} \mathrm{GeV}$, and so on. The interesting thing about this model is that it ties the vacuum energy of the currently active devaluton to the temperature and may explain why the dark energy today is near $T_{0} \sim 3 K$.

Devaluation can easily be generalized within the context of a multidimensional potential, where the minima of the cosine in this paper are replaced by vacua (bowls) of the potential separated by barriers in the multidimensional space. In an upcoming paper [29], we will construct such a scenario in the context of the stringy landscape.

We thank S. Abel, A. Aguirre, M. Cvetič, D. Garfinckel, R. Myers, L. Pando Zayas, D. Spergel and T. Wiseman for helpful discussions. We thank the Michigan Center for Theoretical Physics for hospitality and support while part of this work was completed. This work was supported in part by the US Department of Energy under grant DE-FG02-95ER40899.
[1] A. Riess et al AJ 118, 2675 (1999).

[2] S. Perlmutter etal Ap. J. 517, 565 (1999).

[3] A. H. Guth, Phys. Rev. D 23, 347 (1981).

[4] K. Freese and D. Spolyar, JCAP 0507 (2005) 007.

[5] S. R. Coleman and F. De Luccia, Phys. Rev. D 21, 3305 (1980).

[6] T. Banks, hep-th/0211160

[7] I.K. Affleck and F. De Luccia, Phys. Rev. D 20, 3168 (1979).

[8] P. Sikivie, Phys. Rev. Lett. 48, 1156 (1982).

[9] G. B. Gelmini, M. Gleiser and E. W. Kolb, Phys. Rev. D 39, 1558 (1989).

[10] S.E. Larsson, S. Sarkar, and P.L. White, Phys. Rev. D 55, 5129 (1997).

[11] M. Cvetic, S. Griffies and H. H. Soleng, Phys. Rev. D 48, 2613 (1993) gr-qc/9306005.

[12] M. Cvetic, S. Griffies and H. H. Soleng, Phys. Rev. Lett. 71, 670 (1993) hep-th/9212020.

[13] M. Cvetic and H. H. Soleng, Phys. Rept. 282, 159 (1997) hep-th/9604090.

[14] V. A. Berezin, V. A. Kuzmin and I. I. Tkachev, Phys. Lett. B 120, 91 (1983)

[15] H. Kodama, M. Sasaki, K. Sato and K. i. Maeda, Prog. Theor. Phys. 66, 2052 (1981).

[16] S. K. Blau, E. I. Guendelman and A. H. Guth, Phys. Rev. D 35, 1747 (1987).

[17] E. Farhi, A. H. Guth and J. Guven, Nucl. Phys. B 339, 417 (1990).

[18] A. Aguirre and M. C. Johnson, gr-qc/0508093

[19] L. F. Abbott, Phys. Lett. B 150, 427 (1985).
[20] J. L. Feng, J. March-Russell, S. Sethi and F. Wilczek, Nucl. Phys. B 602, 307 (2001) hep-th/0005276.

[21] J. D. Brown and C. Teitelboim, Nucl. Phys. B 297, 787 (1988).

[22] R. Bousso and J. Polchinski, JHEP 0006, 006 (2000)

[23] E. W. Kolb, D. Seckel and M. S. Turner, Nature 314, 415 (1985).

[24] J. R. Ellis, D. V. Nanopoulos and M. Quiros, Phys. Lett. B 174, 176 (1986).

[25] L. M. Krauss, A. H. Guth, D. N. Spergel, G. B. Field and W. H. Press, Nature 319, 748 (1986).

[26] Y. B. Zeldovich, I. Y. Kobzarev and L. B. Okun, Zh. Eksp. Teor. Fiz. 67, 3 (1974) [Sov. Phys. JETP 40, 1 (1974)].

[27] Z. Lalak, S. Lola, B. Ovrut and G.G. Ross, Nucl. Phys. B 434, 675 (1995).

[28] D. Coulson, Z. Lalak, and B. Ovrut, Phys. Rev. D 53, 4237 (1996).

[29] S. Abel, K. Freese, J. Liu, and D. Spolyar, in preparation.

[30] The opposite behavior is seen for deSitter bubbles, which have $\rho_{G}<0$. The larger the positive vacuum, the smaller the bubble radius needs to be in order for a stable (or dominating) wall to exist.

[31] For completeness, we mention that any remaining high energy regions (which we expect to comprise a minute fraction of the universe) from the exterior point of view look like black holes or wormholes [14, 15, 16, 17, 18]; the former may even contribute to dark matter. 